\title{
Parameterized Complexity of Conflict-Free Matchings and Paths
}

\author{
Akanksha Agrawal \\ Ben-Gurion University of the Negev, Beer-Sheva, Israel \\ agrawal@post.bgu.ac.il
}

\section{Pallavi Jain}

Institute of Mathematical Sciences, HBNI, Chennai, India pallavij@imsc.res.in

\section{Lawqueen Kanesh}

Institute of Mathematical Sciences, HBNI, Chennai, India lawqueen@imsc.res.in

\author{
Saket Saurabh \\ University of Bergen, Bergen, Norway \\ Institute of Mathematical Sciences, HBNI, Chennai, India \\ UMI ReLax \\ saket@imsc.res.in
}

\begin{abstract}
An input to a conflict-free variant of a classical problem $\Gamma$, called CONFLICT-FreE $\Gamma$, consists of an instance $I$ of $\Gamma$ coupled with a graph $H$, called the conflict graph. A solution to ConfLiCT-FreE $\Gamma$ in $(I, H)$ is a solution to $I$ in $\Gamma$, which is also an independent set in $H$. In this paper, we study conflict-free variants of Maximum Matching and Shortest Path, which we call Conflict-Free Matching (CF-Matching) and Conflict-Free Shortest Path (CF-SP), respectively. We show that both CF-MATChing and CF-SP are W[1]-hard, when parameterized by the solution size. Moreover, W[1]-hardness for CF-MATChing holds even when the input graph where we want to find a matching is itself a matching, and W[1]-hardness for CF-SP holds for conflict graph being a unit-interval graph. Next, we study these problems with restriction on the conflict graphs. We give FPT algorithms for CF-MATCHING when the conflict graph is chordal. Also, we give FPT algorithms for both CF-MATCHING and CF-SP, when the conflict graph is $d$-degenerate. Finally, we design FPT algorithms for variants of CF-MATCHING and CF-SP, where the conflicting conditions are given by a (representable) matroid.
\end{abstract}

2012 ACM Subject Classification Theory of computation $\rightarrow$ Parameterized complexity and exact algorithms

Keywords and phrases Conflict-free, Matching, Shortest Path, FPT algorithm, W[1]-hard, Matroid

Digital Object Identifier 10.4230/LIPIcs.MFCS.2019.35

Related Version https://akanksha-agrawal.weebly.com/uploads/1/2/2/2/122276497/cfmatching-shortest_path.pdf

Funding Akanksha Agrawal: PBC Program of Fellowships for Outstanding Post-doctoral Researchers from China and India, reference no. 5101479000.

Pallavi Jain: SERB-NPDF fellowship, reference no. PDF/2016/003508, DST, India.

Saket Saurabh: Horizon 2020 Framework program, the European Research Council (ERC) Consolidator Grant LOPPRE reference no. 819416.

cc) (i) Akanksha Agrawal, Pallavi Jain, Lawqueen Kanesh, and Saket Saurabh; licensed under Creative Commons License CC-BY

44th International Symposium on Mathematical Foundations of Computer Science (MFCS 2019).

Editors: Peter Rossmanith, Pinar Heggernes, and Joost-Pieter Katoen; Article No. 35; pp. 35:1-35:15

Leibniz International Proceedings in Informatics

LIPICS Schloss Dagstuhl - Leibniz-Zentrum für Informatik, Dagstuhl Publishing, Germany 


\section{Introduction}

In the recent years, conflict-free variant of classical combinatorial optimization problems have gained attention from the viewpoint of algorithmic complexity. A typical input to a conflict-free variant of a classical problem $\Gamma$, which we call CONFLICT-FrEE $\Gamma$, consists of an instance $I$ of $\Gamma$ coupled with a graph $H$, called the conflict graph. A solution to Conflict-Free $\Gamma$ in $(I, H)$ is a solution to $I$ in $\Gamma$, which is also an independent set in $H$. Notice that conflict-free version of the problem introduces the constraint of "impossible pairs" in the solution that we seek for. Such a constraint of "impossible pairs" in a solution arises, for example, in the context of program testing and validation [16, 23]. Gabow et al. [16] studied the conflict-free version of paths in a graph, which they showed to be NP-complete.

Conflict-free variants of several classical problems such as, BIN PACKInG [10, 18, 20], Knapsack [35, 32], Minimum Spanning Tree [5, 6], Maximum Matching [6], Maximum Flow [33, 34], Shortest Path [6] and Set Cover [11] have been studied in the literature from the viewpoint of algorithmic complexity, approximation algorithms, and heuristics. It is interesting to note that most of these problems are NP-hard even when their classical counterparts are polynomial time solvable. Recently, Jain et al. [19] and Agrawal et al. [2, 1] initiated the study of conflict-free problems in the realm of parameterized complexity. In particular, they studied CONFLICT-FREE $\mathcal{F}$-DELETION problems for various families $\mathcal{F}$, of graphs such as the family of forests, independent sets, bipartite graphs, interval graphs, etc.

Maximum Matching and Shortest PATH are among the classical graph problems which are of very high theoretical and practical interest. The Maximum Matching problem takes as input a graph $G$, and the objective is to compute a maximum sized subset $Y \subseteq E(G)$ such that no two edges in $Y$ have a common vertex. MAximum MATChing is known to be solvable in polynomial time $[12,28]$. The Shortest PATH problem takes as input a graph $G$ and vertices $s$ and $t$, and the objective is to compute a path between $s$ and $t$ in $G$ with the minimum number of vertices. The SHORTEST PATH problem, together with its variants such as all-pair shortest path, single-source shortest path, weighted shortest path, etc. are known to be solvable in polynomial time [7, 3].

Darmann et al. [6] (among other problems) studied the conflict-free variants of MAXIMUM Matching and Shortest Path. They showed that the conflict-free variant of Maximum MAtChing is NP-hard even when the conflict graph is a disjoint union of edges (matching). Moreover, for the conflict-free variant of SHorTEst PATH, they showed that the problem is APX-hard, even when the conflict graph belongs to the family of 2-ladders.

In this paper, we study the conflict-free versions of matching and shortest path from the viewpoint of parameterized complexity. A parameterized problem $\Pi$ is a subset of $\Sigma^{*} \times \mathbb{N}$, where $\Sigma$ is a fixed, finite alphabet. An instance of a parameterized problem is a pair $(I, k)$, where $I$ is a classical problem instance and $k$ is an integer, which is called the parameter. One of the central notions in parameterized complexity is fixed-parameter tractability, where given an instance $(I, k)$ of a parameterized problem $\Pi$, the goal is to design an algorithm that runs in time $f(k) n^{\mathcal{O}(1)}$, where, $n=|I|$ and $f(\cdot)$ is some computable function, whose value depends only on $k$. An algorithm with running time as described above, is called an FPT algorithm. A parameterized problem that admits an FPT algorithm is said to be in FPT. Not every parameterized problem admits an FPT algorithm, under reasonable complexity-theoretic assumptions. Similar to the notion of NP-hardness and NP-hard reductions in classical Complexity Theory, there are notions of $\mathrm{W}[\mathrm{t}]$-hardness, where $t \in \mathbb{N}$ and parameterized reductions in parameterized complexity. A parameterized problem which is $\mathrm{W}[\mathrm{t}]$-hard, for some $t \in \mathbb{N}$ is believed not to admit an FPT algorithm. For more details on parameterized complexity we refer to the books of Downey and Fellows [9], Flum and Grohe [13], Niedermeier [30], and Cygan et al. [4]. 
Our Results. We study conflict-free (parameterized) variants of MaXimum Matching and Shortest Path, which we call Conflict Free Maximum Matching (CF-MM, for short) and Conflict Free Shortest Path (CF-SP, for short), respectively. These problems are formally defined below.

Conflict Free Maximum Matching (CF-MM)

Parameter: $k$

Input: A graph $G=(V, E)$, a conflict graph $H=\left(E, E^{\prime}\right)$, and an integer $k$.

Question: Is there a matching $M$ of size at least $k$ in $G$, such that $M$ is an independent set in $H$ ?

Conflict Free Shortest Path (CF-SP)

Parameter: $k$

Input: A graph $G=(V, E)$, a conflict graph $H=\left(E, E^{\prime}\right)$, two special vertices $s$ and $t$, and an integer $k$.

Question: Is there an st-path $P$ of length at most $k$ in $G$, such that $E(P)$ is an independent set in $H$ ?

We show that both CF-MM and CF-SP are W[1]-hard, when parameterized by the solution size. The W[1]-hardness for CF-MM is obtained by giving an appropriate reduction from IndEPEndENT SET, which is known to be W[1]-hard, when parameterized by the solution size $[4,8]$. In fact, our W[1]-hardness result for CF-MM holds even when the graph where we want to compute a matching is itself a matching. We show the W[1]-hardness of CF-SP by giving an appropriate reduction from a multicolored variant of the problem UNIT 2-Track INDEPENDENT SET (which we prove to be W[1]-hard). We note that Unit 2-Track InDEPENDENT SET is known to be W[1]-hard, which is used to establish W[1]-hardness of its multicolored variant. We note that our W[1]-hardness result of CF-SP holds even when the conflict graph is a unit interval graph.

Having shown the W[1]-hardness results, we then restrict our attention to having conflict graphs belonging to some families of graphs, where the InDEPEnDENT SET problem is either polynomial time solvable or solvable in FPT time. Two of the very well-known graph families that we consider are the family of chordal graphs and the family of $d$-degenerate graphs. For the CF-MM problem, we give an FPT algorithm, when the conflict graph belongs to the family of chordal graphs. Our algorithm is based on a dynamic programming over a "structured" tree decomposition of the conflict graph (which is chordal) together with "efficient" computation of representative families at each step of our dynamic programming routine. Notice that we cannot obtain an FPT algorithm for the CF-SP problem when the conflict graph is a chordal graph. This holds because unit-interval graphs are chordal, and the problem CF-SP is W[1]-hard, even when the conflict graph is a unit-interval graph.

For conflict graphs being $d$-degenerate, we obtain FPT algorithms for both CF-MM and CF-SP. These algorithms are based on the computation of an independence covering family, a notion which was recently introduced by Lokshtanov et al. [25]. We note that even for nowhere dense graphs, such an independence covering family can be computed efficiently [25]. Since our algorithms are based on computation of independence covering families, hence, our results hold even when the conflict graph is a nowhere dense graph.

Finally, we study a variant of CF-MM and CF-SP, where instead of conflicting conditions being imposed by independent sets in a conflict graph, they are imposed by independence constraints in a (representable) matroid. We give FPT algorithms for the above variant of both CF-MM and CF-SP.

Due to space limitations, many proofs have been omitted from the extended abstract. 


\section{Preliminaries}

Sets and graph notations. For $n \in \mathbb{N}$, by $[n]$ and $[0, n]$, we denote the sets $\{1,2, \cdots, n\}$ and $\{0,1,2, \cdots, n\}$, respectively. For a set $U$ and $p \in \mathbb{N}$, a $p$-family (over $U$ ) is a family of subsets of $U$ of size $p$. We let $\omega$ denote the exponent in the running time of algorithm for matrix multiplication, the current best known bound for it is $\omega<2.373$ [36]. Consider a graph $G$. For $X \subseteq V(G), G[X]$ denotes the subgraph of $G$ with vertex set $X$ and edge set $\{u v \in E(G) \mid u, v \in X\}$. For $Y \subseteq E(G), G[Y]$ denotes the subgraph of $G$ with vertex set $\cup_{u v \in Y}\{u, v\}$ and edge set $Y$.

We define a structured tree decomposition that will be used in our algorithm.

- Definition 1 ([4, 22]). Let $(T, X)$ be a tree decomposition of a graph $H$ with $r$ as the root node. That is, $T$ is a tree rooted at $r$ and $X=\left\{X_{t} \mid t \in V(T)\right\}$. Then, $(T, X)$ is a nice tree decomposition if for each each leaf $\ell$ in $T$ and the root $r$, we have that $X_{\ell}=X_{r}=\emptyset$, and each non-leaf node $t \in V(T)$ is of one of the following types: Introduce node: $t$ has exactly one child, say $t^{\prime}$, and $X_{t}=X_{t^{\prime}} \cup\{v\}$, where $v \notin X_{t^{\prime}}$. We say that $v$ is introduced at $t$; Forget node: $t$ has exactly one child, say $t^{\prime}$, and $X_{t}=X_{t^{\prime}} \backslash\{v\}$, where $v \in X_{t^{\prime}}$. We say that $v$ is forgotten at $t$; Join node: $t$ has exactly two children, say $t_{1}$ and $t_{2}$, and $X_{t}=X_{t_{1}}=X_{t_{2}}$.

A tree decomposition $(T, X)$ of a graph $H$, where for each $t \in V(T)$, the graph $H\left[X_{t}\right]$ is a clique, is called a clique-tree. The result below follows from existence of clique-tree for chordal graphs [17] and an algorithm for computation of a nice tree decomposition [4, 22].

- Proposition 2. Given an $n$ vertex chordal graph $H$, in polynomial time we can construct a nice tree decomposition which is also a clique-tree (nice clique-tree), $(T, X)$ of $H$ with $\mathcal{O}\left(n^{2}\right)$ nodes.

Matroids and representative sets. In the following we state some definitions related to matroids used in the paper. We refer the reader to [31] for more details. We also state the definition of representative families and state some results related to them.

- Definition $3([4,31])$. A matroid $\mathcal{M}=(U, \mathcal{I})$ is a partition matroid if the ground set $U$ is partitioned into sets $U_{1}, U_{2}, \cdots, U_{k}$, and for each $i \in[k]$, there is an integer $a_{i}$ associated with $U_{i}$. A set $S \subseteq U$ is an independent in $\mathcal{M}$ if and only if for each $i \in[k],\left|S \cap U_{i}\right| \leq a_{i}$.

- Proposition $4([15,31,26])$. A representation of a partition matroid over $\mathbb{Q}$ (the field of rationals) can be computed in polynomial time.

- Definition 5. Let $\mathcal{M}_{1}=\left(U_{1}, \mathcal{I}_{1}\right), \mathcal{M}_{2}=\left(U_{2}, \mathcal{I}_{2}\right) \cdots, \mathcal{M}_{t}=\left(U_{t}, \mathcal{I}_{t}\right)$ be $t$ matroids with $U_{i} \cap U_{j}=\emptyset$, for all $1 \leq i \neq j \leq t$. The direct sum $\mathcal{M}_{1} \oplus \cdots \oplus \mathcal{M}_{t}$, of $\mathcal{M}_{1}, \mathcal{M}_{2}, \cdots, \mathcal{M}_{t}$ is the matroid with ground set $U=\cup_{i \in[t]} U_{i}$ and $X \subseteq U$ is independent in $\mathcal{M}$ if and only if for each $i \in[t], X \cap U_{i} \in \mathcal{I}_{i}$.

- Proposition 6 ([27, 31]). Given matrices $A_{1}, A_{2}, \cdots, A_{t}$ (over $\mathbb{F}$ ) representing matroids $\mathcal{M}_{1}, \mathcal{M}_{2}, \cdots, \mathcal{M}_{t}$, respectively, we can compute a representation of their direct sum, $\mathcal{M}_{1} \oplus$ $\cdots \oplus \mathcal{M}_{t}$, in polynomial time.

Next, we state the definition of representative families.

- Definition 7. Let $\mathcal{M}=(U, \mathcal{I})$ be a matroid, and $\mathcal{A}$ be a $p$-family of $U$. We say that $\mathcal{A}^{\prime} \subseteq \mathcal{A}$ is a $q$-representative for $\mathcal{A}$ if for every set $Y \subseteq U$ of size $q$, if there is a set $X \in \mathcal{A}$, such that $X \cap Y=\emptyset$ and $X \cup Y \in \mathcal{I}$, then there is a set $X^{\prime} \in \mathcal{A}^{\prime}$ such that $X^{\prime} \cap Y=\emptyset$ and $X^{\prime} \cup Y \in \mathcal{I}$. If $\mathcal{A}^{\prime} \subseteq \mathcal{A}$ is a $q$-representative for $\mathcal{A}$ then we denote it by $\mathcal{A}^{\prime} \subseteq{ }_{\text {rep }}^{q} \mathcal{A}$. 
- Theorem $8([4,14])$. Given a matrix $M$ (over field $\mathbb{F}$ ) representing a matroid $\mathcal{M}=(U, \mathcal{I})$ of rank $k$, a p-family $\mathcal{A}$ of independent sets in $\mathcal{M}$, and an integer $q$ such that $p+q=k$, there is an algorithm which computes a q-representative family $\mathcal{A}^{\prime} \subseteq$ rep $\mathcal{A}$ of size at most $\left(\begin{array}{c}p+q \\ p\end{array}\right)$ using at most $\mathcal{O}\left(|\mathcal{A}|\left(\left(\begin{array}{c}p+q \\ p\end{array}\right) p^{\omega}+\left(\begin{array}{c}p+q \\ p\end{array}\right)^{\omega-1}\right)\right)$ operations over $\mathbb{F}$.

Let $\mathcal{A}_{1}$ and $\mathcal{A}_{2}$ be two families of sets over $U$ and $\mathcal{M}=(U, \mathcal{I})$ be a matroid. We define their convolution as: $\mathcal{A}_{1} \star \mathcal{A}_{2}=\left\{A_{1} \cup A_{2} \mid A_{1} \in \mathcal{A}_{1}, A_{2} \in \mathcal{A}_{2}, A_{1} \cap A_{2}=\emptyset\right.$ and $\left.A_{1} \cup A_{2} \in \mathcal{I}\right\}$.

Universal sets and their computation. An $(n, k)$-universal set is a family $\mathcal{F}$ of subsets of $[n]$ such that for any set $S \subseteq[n]$ of size $k$, the family $\{A \cap S \mid A \in \mathcal{F}\}$ contains all $2^{k}$ subsets of $S$.

- Proposition 9 ([4, 29]). For any $n, k \geq 1$, we can compute an $(n, k)$-universal set of size $2^{k} k^{\mathcal{O}(\log k)} \log n$ in time $2^{k} k^{\mathcal{O}(\log k)} n \log n$.

\section{W[1]-hardness Results}

In this section, we show that Conflict Free Maximum Matching and Conflict Free ShorTest PATH are W[1]-hard, when parameterized by the solution size.

W[1]-hardness of CF-MM. We show that CF-MM is W[1]-hard, when parameterized by the solution size, even when the graph where we want to find a matching, is itself a matching (disjoint union of edges). To prove our result, we give an appropriate reduction from Independent SET to CF-MM. It is known that Independent Set is W[1]-hard, when parameterized by the size of an independent set $[4,8]$. Given an instance $\left(G^{\star}, k\right)$ of InDEPENDENT SET, we construct an equivalent instance $(G, H, k)$ of $\mathrm{CF}-\mathrm{MM}$ as follows. We first describe the construction of $G$. For each $v \in V\left(G^{\star}\right)$, we add an edge $v v^{\prime}$ to $G$. Notice that $G$ is a matching. This completes the description of $G$. Next, we move to the construction of $H$. We have $V(H)=\left\{e_{v}=v v^{\prime} \mid v \in V\left(G^{\star}\right)\right\}$. Moreover, for $e_{u}, e_{v} \in V(H)$, we add the edge $e_{u} e_{v}$ to $E(H)$ if and only if $u v \in E\left(G^{\star}\right)$. We note that $H$ is exactly the same as $G^{\star}$, with vertices being renamed. This completes the description of the reduction. We obtain the following theorem from the equivalence of instances $\left(G^{\star}, k\right)$ of INDEPENDENT SET and $(G, H, k)$ of CF-MM.

- Theorem 10. CF-MM is W[1]-hard, when parameterized by the solution size.

W[1]-hardness of CF-SP. We show that CF-SP is W[1]-hard, when parameterized by the solution size, even when the conflict graph is a proper interval graph. We refer to this restricted variant of the problem as UNIT INTERVAL CF-SP. To prove our result, we give an appropriate reduction from a multicolored variant of the problem UNIT 2-TRACK Independent Set, which we call Unit 2-Track Multicolored IS. In the following, we define the problem Unit 2-TraCK Multicolored IS.

Unit 2-Track Multicolored IS (Unit 2-Track MIS)

Parameter: $k$

Input: Two unit-interval graphs $G_{1}=\left(V, E_{1}\right)$ and $G_{2}=\left(V, E_{2}\right)$, and a partition $V_{1}, V_{2}, \cdots, V_{k}$ of $V$.

Question: Is there a set $S \subseteq V$, such that $S$ is an independent set in both $G_{1}$ and $G_{2}$, and for each $i \in[k]$, we have $\left|S \cap V_{i}\right|=1$ ? 
It is known that UNIT 2-TRACK IS is W[1]-hard, when parameterized by the solution size [21]. We can show that the problem UNIT 2-TRACK MIS is W[1]-hard, when parameterized by the number of sets in the partition by giving an appropriate (Turing) reduction from Unit 2-Track IS. We give a reduction from Unit 2-Track MIS to Unit InTERVAL CF-SP, and hence obtaining the desired result.

We now give a parameterized reduction from UNIT 2-TRACK MIS to UNIT INTERVAL CF-SP. Let $\left(G_{1}, G_{2}, V_{1}, \cdots, V_{k}\right)$ be an instance of Unit 2-Track MIS. We construct an instance $\left(G^{\prime}, H, s, t, k^{\prime}\right)$ of Unit Interval CF-SP as follows. For each $v \in V\left(G_{1}\right)$, we add a path on 3 vertices namely, $\left(v_{1}, v_{2}, v_{3}\right)$ in $G^{\prime}$. For notational convenience, for $v \in V\left(G_{1}\right)$, by $e_{12}(v)$ and $e_{23}(v)$ we denote the edges $v_{1} v_{2}$ and $v_{2} v_{3}$, respectively. Consider $i \in[k-1]$. For $u \in V_{i}$ and $v \in V_{i+1}$, we add the edge $z_{u v}=u_{3} v_{1}$ to $E\left(G^{\prime}\right)$. Moreover, by $Z_{i}$, we denote the set $\left\{z_{u v} \mid u \in V_{i}, v \in V_{i+1}\right\}$. We add two new vertices $s$ and $t$ to $V\left(G^{\prime}\right)$, and add all the edges in $Z_{0}=\left\{s v_{1} \mid v \in V_{1}\right\}$ and $Z_{k}=\left\{v_{3} t \mid v \in V_{k}\right\}$ to $E\left(G^{\prime}\right)$. Next, we move to the construction of $H$. Note that $H$ must be a unit-interval graph on the vertex set $E\left(G^{\prime}\right)=\left(\cup_{i \in[0, k]} Z_{i}\right) \cup\left(\cup_{v \in V\left(G_{1}\right)}\left\{e_{12}(v), e_{23}(v)\right\}\right)$. In $H$, each vertex in $\cup_{i \in[0, k]} Z_{i}$ is an isolated vertex. Let $E_{12}=\left\{e_{12}(v) \mid v \in V\left(G_{1}\right)\right\}$ and $E_{23}=\left\{e_{23}(v) \mid v \in V\left(G_{1}\right)\right\}$. For $e_{12}(u), e_{12}(v) \in E_{12}$, we add the edge $e_{12}(u) e_{12}(v)$ to $E(H)$ if and only if $u v \in E\left(G_{1}\right)$. Similarly, for $e_{23}(u), e_{23}(v) \in E_{23}$, we add the edge $e_{23}(u) e_{23}(v)$ to $E(H)$ if and only if $u v \in E\left(G_{2}\right)$. Observe that $H\left[E_{12}\right]$ is isomorphic to $G_{1}$, with bijection $\phi_{1}: V\left(G_{1}\right) \rightarrow E_{12}$ with $\phi_{1}(v)=e_{12}(v)$. Similarly, $H\left[E_{23}\right]$ is isomorphic to $G_{2}$ with bijection $\phi_{2}: V\left(G_{2}\right) \rightarrow E_{23}$ with $\phi_{2}(v)=e_{23}(v)$. By construction, $H$ is a disjoint union of unit-interval graphs, and hence is a unit-interval graph. Finally, we set $k^{\prime}=3 k+1$. This completes the description of the reduction. We obtain the following theorem from the equivalence of instances $\left(G_{1}, G_{2}, V_{1}, \cdots, V_{k}\right)$ of Unit 2-Track MIS and $\left(G^{\prime}, H, s, t, k^{\prime}\right)$ of Unit InTERVAL CF-SP.

- Theorem 11. Unit Interval CF-SP is W[1]-hard, when parameterized by the solution size.

\section{FPT Algorithm for CF-MM with Chordal Conflict}

In this section, we show that CF-MM is FPT, when the conflict graph is a chordal graph. We call this restricted version of CF-MM as Chordal Conflict Matching. Towards designing an algorithm for Chordal Conflict Matching, we first give an FPT algorithm for a restricted version of CHORDAL CONFLICT MATCHING, where the graph where we want to compute a matching is a bipartite graph. We call this variant of CHORDAL ConfLICT Matching as Chordal Conflict Bipartite Matching (CCBM). We then employ the algorithm for CCBM to design an FPT algorithm for Chordal Conflict Matching.

\subsection{FPT algorithm for CCBM}

We design an FPT algorithm for the problem CCBM, where the conflict graph is chordal and the graph where we want to compute a matching is a bipartite graph. The problem CCBM is formally defined below.

Chordal Conflict Bipartite Matching (CCBM)

Parameter: $k$

Input: A bipartite graph $G=(V, E)$ with vertex bipartition $L, R$, a conflict graph $H=\left(E, E^{\prime}\right)$, and an integer $k$.

Question: Is there a matching $M \subseteq E$ of size $k$ in $G$, such that $M$ is an independent set in $H$ ? 
The FPT algorithm for CCBM is based on a dynamic programming routine over tree decomposition of the conflict graph $H$ and representative sets on the graph $G$. Let $(G, L, R, H, k)$ be an instance of CF-MM, where $G$ is a bipartite graph on $n$ vertices, with vertex bipartition $L, R$, and $H$ is a chordal graph with $V(H)=E(G)$.

In the following, we construct three matroids $\mathcal{M}_{L}=\left(E, \mathcal{I}_{L}\right), \mathcal{M}_{R}=\left(E^{c}, \mathcal{I}_{R}\right)$, and $\mathcal{M}=\left(E \cup E^{c}, \mathcal{I}\right)$. Matroids $\mathcal{M}_{L}$ and $\mathcal{M}_{R}$ are partition matroids and the matroid $\mathcal{M}$ is the direct sum of $\mathcal{M}_{L}$ and $\mathcal{M}_{R}$. The ground set of $\mathcal{M}_{L}$ is $E=E(G)$. The set $E^{c}$ contains a copy of edges in $E$, i.e., $E^{c}=\left\{e^{c} \mid e \in E\right\}$. We create two (disjoint) sets $E$ and $E^{c}$, because $\mathcal{M}$ is the direct sum of $\mathcal{M}_{L}$ and $\mathcal{M}_{R}$, and we want their ground sets to be disjoint. Next, we describe the partition $\mathcal{E}$ of $E$ into $|L|$ sets and $|L|$ integers, one for each set in the partition, for the partition matroid $\mathcal{M}_{L}$. For $u \in L$, let $E_{u}=\{u v \mid u v \in E\}$. Notice that for $u, v \in L$, where $u \neq v$, we have $E_{u} \cap E_{v}=\emptyset$. Moreover, $\cup_{u \in E} E_{u}=E$. We let $\mathcal{E}=\left\{E_{u} \mid u \in L\right\}$, and for each $u \in L$, we set $a_{u}=1$. Similarly, we define the partition $\mathcal{E}^{c}$ of $E^{c}$ with respect to set $R$. That is, we let $\mathcal{E}^{c}=\left\{E_{u}^{c}=\left\{(u v)^{c} \mid u v \in E(G)\right\} \mid u \in R\right\}$. Furthermore, for $u \in R$, we let $a_{u^{c}}=1$. We define the following notation, which will be used later. For $Z \subseteq E$, we let $Z^{c}=\left\{e^{c} \mid e \in Z\right\} \subseteq E^{c}$.

To capture the independence property on the conflict graph, we rely on the fact that a chordal graph admits a nice clique-tree (Proposition 2). This allows us to do dynamic programming over a nice clique-tree. At each step of our dynamic programming routine, using representative sets, we ensure that we store a family of sets which are enough to recover (some) independent set in $\mathcal{M}$, if a solution exists.

We now move to the formal description of the algorithm. The algorithm starts by computing a nice clique-tree $(T, X)$ of $H$ in polynomial time, using Proposition 2 . Let $r \in V(T)$ be the root of the (rooted) tree $T$. For $X_{t} \in X$, we let $\mathcal{X}_{t}=\{\emptyset\} \cup\left\{\{v\} \mid v \in X_{t}\right\}$. For a node $t \in V(T)$, by $\operatorname{desc}(t)$ we denote the set descendant of $t$ in $T$ (including $t$ ). For $t \in V(T)$, $H_{t}$ is the graph $H\left[V_{t}\right]$, where $V_{t}=\cup_{d \in \operatorname{desc}(t)} X_{d}$.

In the following, we state some notations, which will be used in the algorithm. For each $t \in V(T), Y \in \mathcal{X}_{t}$, and an integer $p \in[0, k]$ we define a family $\mathcal{P}_{t, Y}^{p}$ as: $\mathcal{P}_{t, Y}^{p}=\left\{Z \cup Z^{c}\right.$ $Z \subseteq V\left(H_{t}\right)(\subseteq E),|Z|=p, Z \cap X_{t}=Y, Z \cup Z_{c} \in \mathcal{I}$ and $H_{t}[Z]$ is edgeless $\}$. For a family $\mathcal{F}$ of subsets of $E \cup E^{c}, \mathcal{F}$ is called a paired-family if for each $F \in \mathcal{F}$, there is $Z \subseteq E$, such that $F=Z \cup Z^{c}$. Next, we state the entries in our dynamic programming routine.

- Definition 12. For each $t \in V(T), Y \in \mathcal{X}_{t}$ and $p \in[0, k]$, we have an entry $c[t, Y, p]$, which stores a paired-family $\mathcal{F}(t, Y, p)$ of subsets of $E \cup E^{c}$ of size $2 p$, such that for each $F=Z \cup Z^{c} \in \mathcal{F}$, the following conditions are satisfied: $|Z|=p ; Z \cap X_{t}=Y ; Z$ is a matching in $G$, i.e., $Z$ and $Z^{c}$ are independent sets in $\mathcal{M}_{L}$ and $\mathcal{M}_{R}$, respectively; $Z$ is an independent set in $H_{t}$. Moreover, $\mathcal{F} \neq \emptyset$ if and only if $\mathcal{P}_{t, Y}^{p} \neq \emptyset$.

Consider $t \in V(T), Y \in \mathcal{X}_{t}$ and $p \in[0, k]$. Observe that $\mathcal{P}_{t, Y}^{p}$ is a valid candidate for $c[t, Y, p]$, which also implies that $(G, H, k)$ is a yes instance of CCBM if and only if $c[r, \emptyset, k] \neq \emptyset$. However, we cannot set $c[t, Y, p]=\mathcal{P}_{t, Y}^{p}$ as the size of $\mathcal{P}_{t, Y}^{p}$ could be exponential in $n$, and the goal here is to obtain an FPT algorithm. Hence, we will store a much smaller subfamily (of size at most $\left(\begin{array}{l}2 k \\ 2 p\end{array}\right)$ ) of $\mathcal{P}_{t, Y}^{p}$ in $c[t, Y, p]$, which will be computed using representative sets. Moreover, as we have a structured form of a tree decomposition (nice clique-tree) of $H$, we compute the entries of the table based on the entries of its children, which will be given by recursive formulae. For leaf nodes, which form base cases for recursive formulae, we compute all entries directly.

Next, we give (recursive) formulae for the computation of the table entries. Consider $t \in V(T), Y \in \mathcal{X}_{t}$ and $p \in[0, k]$. We compute the entry $c[t, Y, k]$ based on the following cases. 
Leaf node. $t$ is a leaf node. In this case, we have $X_{t}=\emptyset$, and hence $\mathcal{X}_{t}=\{\emptyset\}$. If $p=0$, then $\mathcal{P}_{t, \emptyset}^{p}=\{\emptyset\}$, and $\mathcal{P}_{t, \emptyset}^{p}=\emptyset$, otherwise. Since, $\mathcal{P}_{t, \emptyset}^{p}$ is a valid candidate for $c[t, Y, p]$, we set $c[t, Y, p]=\mathcal{P}_{t, \emptyset}^{p}$. Note that $c[t, Y, p]$ has size at most $1 \leq\left(\begin{array}{l}2 k \\ 2 p\end{array}\right)$, and we can compute $c[t, Y, p]$ in polynomial time.

Introduce node. Suppose $t$ is an introduce node with child $t^{\prime}$ such that $X_{t}=X_{t^{\prime}} \cup\{e\}$, where $e \notin X_{t^{\prime}}$. If $Y \neq \emptyset$ and $p<1$, then we set $c[t, Y, p]=\emptyset$. Otherwise, we compute the entry as described below. Before computing the entry $c[t, Y, p]$, we first compute a set $\widetilde{\mathcal{P}}_{t, Y}^{p}$ as follows. If $Y \neq\{e\}$, then we set $\widetilde{\mathcal{P}}_{t, Y}^{p}=c\left[t^{\prime}, Y, p\right]$, and otherwise, $\widetilde{\mathcal{P}}_{t, Y}^{p}=c\left[t^{\prime}, \emptyset, p-1\right] \star\left\{\left\{e, e^{c}\right\}\right\}$.

Next, we compute $\widehat{\mathcal{P}}_{t, Y}^{p} \subseteq_{\text {rep }}^{2 k-2 p} \widetilde{\mathcal{P}}_{t, Y}^{p}$ of size $\left(\begin{array}{l}2 k \\ 2 p\end{array}\right)$, using Theorem 8 . Finally, we set $c[t, Y, p]=\widehat{\mathcal{P}}_{t, Y}^{p}$.

Forget node. Suppose $t$ is a forget node with child $t^{\prime}$ such that $X_{t}=X_{t^{\prime}} \backslash\{e\}$, where $e \in X_{t^{\prime}}$. Before computing the entry $c[t, Y, p]$, we first compute a set $\widetilde{\mathcal{P}}_{t, Y}^{p}$ as follows. If $Y \neq \emptyset$, we set $\widetilde{\mathcal{P}}_{t, Y}^{p}=c\left[t^{\prime}, Y, p\right]$, and otherwise, $\widetilde{\mathcal{P}}_{t, Y}^{p}=c\left[t^{\prime}, \emptyset, p\right]$.

Next, we compute $\widehat{\mathcal{P}}_{t, Y}^{p} \subseteq_{\text {rep }}^{2 k-2 p} \widetilde{\mathcal{P}}_{t, Y}^{p}$ of size $\left(\begin{array}{l}2 k \\ 2 p\end{array}\right)$, using Theorem 8 . Finally, we set $c[t, Y, p]=\widehat{\mathcal{P}}_{t, Y}^{p}$.

Join node. Suppose $t$ is a join node with children $t_{1}$ and $t_{2}$, such that $X_{t}=X_{t_{1}}=X_{t_{2}}$. If $Y \neq \emptyset$ and $p<1$, then we set $c[t, Y, p]=\emptyset$. Otherwise, we compute the entry as described below. Before computing the entry $c[t, Y, p]$, we first compute a set $\widetilde{\mathcal{P}}_{t, Y}^{p}$ as follows. If $Y=\emptyset$, we set $\tilde{\mathcal{P}}_{t, Y}^{p}=\cup_{i \in[0, p]}\left(c\left[t_{1}, \emptyset, i\right] \star c\left[t_{2}, \emptyset, p-i\right]\right)$, and otherwise, $\tilde{\mathcal{P}}_{t, Y}^{p}=$ $\cup_{i \in[p]}\left(c\left[t_{1}, Y, i\right] \star c\left[t_{2}, \emptyset, p-i\right]\right)$.

Next, we compute $\widehat{\mathcal{P}}_{t, Y}^{p} \subseteq_{\text {rep }}^{2 k-2 p} \widetilde{\mathcal{P}}_{t, Y}^{p}$ of size $\left(\begin{array}{l}2 k \\ 2 p\end{array}\right)$, using Theorem 8 . Finally, we set $c[t, Y, p]=\widehat{\mathcal{P}}_{t, Y}^{p}$.

This completes the description of the (recursive) formulae for computing all entries of the table. The correctness of the algorithm follows from the correctness of the (recursive) formulae, and the fact that $(G, H, k)$ is a yes instance of CCBM if and only if $c[r, \emptyset, k] \neq \emptyset$. From the above, we obtain the following theorem.

- Theorem 13. CCBM admits an FPT algorithm running in time $\mathcal{O}\left(2^{\mathcal{O}(\omega k)} n^{\mathcal{O}(1)}\right)$.

\subsection{FPT algorithm for Chordal Conflict Matching}

We design an FPT algorithm for CHORDAL CONFLICT MATCHING, using the algorithm for CCBM (Theorem 13). Let $(G, H, k)$ be an instance of CF-MM, where $H$ is a chordal graph and $G$ is a graph on $n$ vertices. We assume that $G$ is a graph on vertex set $[n]$, which can easily be achieved by renaming vertices.

The algorithm starts by computing an $(n, 2 k)$-universal set $\mathcal{F}$, using Proposition 9 . For each set $A \in \mathcal{F}$, the algorithm constructs an instance $I_{A}=\left(G_{A}, L_{A}, R_{A}, H_{A}, k\right)$ of CCBM as follows. We have $V\left(G_{A}\right)=V(G), L_{A}=A, R=V(G) \backslash A, E\left(G_{A}\right)=\{u v \in E(G) \mid u \in$ $\left.L_{A}, v \in R_{A}\right\}$, and $H_{A}=H\left[E\left(G_{A}\right)\right]$. Note that $H_{A}$ is a chordal graph because chordal graphs are closed under induced subgraphs and disjoint unions. The algorithm decides the instance $I_{A}$ using Theorem 13, for each $A \in \mathcal{F}$. The algorithm outputs yes if and only if there is $A \in \mathcal{F}$, such that $I_{A}$ is a yes instance of CCBM. 
- Theorem 14. The algorithm presented for CF-MM is correct, Moreover, it runs in time $2^{\mathcal{O}(\omega k)} k^{\mathcal{O}(\log k)} n^{\mathcal{O}(1)}$, where $\omega<2.373$ is the exponent of matrix multiplication and $n$ is the number of vertices in the input graph.

Proof. Let $(G, H, k)$ be an instance of CF-MM, where $H$ is a chordal graph and $G$ is a graph on vertex set $[n]$. Clearly, if the algorithm outputs yes, then indeed $(G, H, k)$ is a yes instance of CF-MM. Next, we argue that if $(G, H, k)$ is a yes instance of CF-MM then the algorithm returns yes. Suppose there is a solution $M \subseteq E(G)$ to $\mathrm{CF}-\mathrm{MM}$ in $(G, H, k)$. Let $S=\{i, j \mid i j \in M\}$, and $L=\{i \mid$ there is $j \in[n]$ such that $i j \in M$ and $i<j\}$. Observe that $|S|=2 k$. Since $\mathcal{F}$ is an $(n, 2 k)$-universal set, there is $A \in \mathcal{F}$ such that $A \cap S=L$. Note that $S$ is a solution to CCBM in $I_{A}$. This together with Theorem 13 implies that the algorithm will return yes as output.

Next, we prove the claimed running time of the algorithm. The algorithm computes $(n, 2 k)$ universal set of size $\mathcal{O}\left(2^{2 k} k^{\mathcal{O}(\log k)} \log n\right)$, in time $\mathcal{O}\left(2^{2 k} k^{\mathcal{O}(\log k)} n \log n\right)$, using Proposition 9 . Then for each $A \in \mathcal{F}$, the algorithm creates an instance $I_{A}$ of CCBM in polynomial time. Furthermore, it resolves the $I_{A}$ of CCBM in time $\mathcal{O}\left(2^{\mathcal{O}(\omega k)} n^{\mathcal{O}(1)}\right)$ using Theorem 13. Hence, the running time of the algorithm is bounded by $2^{\mathcal{O}(\omega k)} k^{\mathcal{O}(\log k)} n^{\mathcal{O}(1)}$.

\section{FPT algorithms for CF-MM and CF-SP with matroid constraints}

In this section, we study the problems CF-MM and CF-SP, where the conflicting condition is being an independent set in a (representable) matroid. Due to technical reasons we only consider the case when the matroid is representable over $\mathbb{Q}$ (the field of rationals).

\subsection{FPT algorithm for Matroid CF-MM}

We study a variant of the problem CF-MM, where the conflicting condition is being an independent set in a matroid representable over $\mathbb{Q}$. We call this variant of $\mathrm{CF}-\mathrm{MM}$ as Rational Matroid CF-MM (RAT MAT CF-MM, for short), which is formally defined below.

Rational Matroid CF-MM (RAt Mat CF-MM) Parameter: $k$

Input: A graph $G$, a matrix $A_{\mathcal{M}}$ (representing a matroid $\mathcal{M}$ over $\mathbb{Q}$ ) with columns indexed by $E(G)$, and an integer $k$.

Question: Is there a matching $M \subseteq E(G)$ of size at most $k$, such that the set of columns in $M$ are linearly independent (over $\mathbb{Q})$ ?

We design an FPT algorithm for RAT MAT CF-MM. Towards designing an algorithm for RAT MAT CF-MM, we first give an FPT algorithm for a restricted version of RAT MAT CF-MM, where the graph in which we want to compute a matching is a bipartite graph. We call this variant of RAt MAT CF-MM as RAt Mat CF-Bipartite Matching (RAT Mat CF-BM). We then employ the algorithm for RAT MAT CF-BM to design an FPT algorithm for RAT MAT CF-MM.

\subsubsection{FPT algorithm for Rat Mat CF-BM}

We design an FPT algorithm for the problem RAT MAT CF-BM, where the conflicting condition is being an independent set in a matroid (representable over $\mathbb{Q}$ ) and the graph where we want to compute a matching is a bipartite graph.

Our algorithm takes an instance of RAT MAT CF-BM and generates an instance of 3-MATROID InTERSECTION, and then employs the known algorithm for 3-MATROID 
INTERSECTION to resolve the instance. In the following, we formally define the problem 3-MATROId InTERSECTION.

3-MATROID INTERSECTION

Parameter: $k$

Input: Matrices $A_{\mathcal{M}_{1}}, A_{\mathcal{M}_{2}}$, and $A_{\mathcal{M}_{3}}$ over $\mathbb{F}$ (representing matroids $\mathcal{M}_{1}, \mathcal{M}_{2}$, and $\mathcal{M}_{3}$, respectively, on the same ground set $E$ ) with columns indexed by $E$, and an integer $k$.

Question: Is there a set $M \subseteq E$ of size $k$, such that $M$ is independent in each $\mathcal{M}_{i}$, for $i \in[3]$ ?

Before moving further, we briefly explain why we needed an additional constraint that the input matrix is representable over $\mathbb{Q}$. Firstly, we will be using partition matroids which are representable only on fields of large enough size, and we want all the matroids, i.e. the one which is part of the input and the ones that we create, to be representable over the same field. Secondly, the algorithmic result (with the desired running time) we use for 3-MATROID INTERSECTION works only for certain types of fields.

Next, we state an algorithmic result regarding 3-MATROID INTERSECTION [24], which is be used by the algorithm. We note that we only state a restricted form of the algorithmic result for 3-MATROID INTERSECTION in [24], which is enough for our purpose.

- Proposition 15 (Proposition 4.8 [24] (partial)). 3-MATROID INTERSECTION can be solved in $\mathcal{O}\left(2^{3 \omega k}\left\|A_{M}\right\|^{\mathcal{O}(1)}\right)$ time, when the matroids are represented over $\mathbb{Q}$.

We are now ready to prove the desired result.

- Theorem 16. Rat MAT CF-BM can be solved in $\mathcal{O}\left(2^{3 \omega k}\left\|A_{M}\right\|^{\mathcal{O}(1)}\right)$ time.

Proof. Let $\left(G=(V, E), L, R, A_{\mathcal{M}}, k\right)$ be an instance of RAT MAT CF-BM, where the matrix $A_{\mathcal{M}}$ represents a matroid $\mathcal{M}=(E, \mathcal{I})$ over $\mathbb{Q}$ and $L, R$ is a partition of $V$ into independent sets. Let $\mathcal{M}_{L}=\left(E, \mathcal{I}_{L}\right), \mathcal{M}_{R}=\left(E, \mathcal{I}_{R}\right)$ be the partition matroids as defined in Section 4. Next we compute matrix representations $A_{\mathcal{M}_{L}}$ and $A_{\mathcal{M}_{R}}$ of matroids $\mathcal{M}_{L}, \mathcal{M}_{R}$, respectively, using Proposition 4. Now, we solve 3-MATROID InTERSECTION on the instance $\left(\mathcal{M}, A_{\mathcal{M}_{L}}, A_{\mathcal{M}_{R}}, k\right)$ (over $\left.\mathbb{Q}\right)$ using Proposition 15, and return the same answer, as returned by the algorithm in it. The correctness follows directly from the following. $S \subseteq E$ is a matching in $G$ if and only if $S$ is an independent set in $\mathcal{M}_{L}$ and $\mathcal{M}_{R}$, that is $S \in \mathcal{I}_{L} \cap \mathcal{I}_{R}$. The claimed running time follows from Proposition 4 and Proposition 15.

\subsubsection{FPT algorithm for Rat Mat CF-MM}

We design an FPT algorithm for RAT MAT CF-MM, using the algorithm for RAT MAT CF-BM (Theorem 13). Let $\left(G, A_{\mathcal{M}}, k\right)$ be an instance of RAT MAT CF-MM, where the matrix $A_{\mathcal{M}}$ represents a matroid $\mathcal{M}=(E, \mathcal{I})$ over $\mathbb{Q}$. We assume that $G$ is a graph with the vertex set $[n]$, which can easily be achieved by renaming vertices.

The algorithm starts by computing an $(n, 2 k)$-universal set $\mathcal{F}$, using Proposition 9 . For each set $X \in \mathcal{F}$, the algorithm constructs an instance $I_{X}=\left(G_{X}, L_{X}, R_{X}, A_{\mathcal{M}}, k\right)$ of RAT MAt CF-BM as follows. We have $V\left(G_{X}\right)=V(G), L_{X}=X, R=V(G) \backslash X$, $E\left(G_{X}\right)=\left\{u v \in E(G) \mid u \in L_{X}, v \in R_{X}\right\}$. The algorithm decides the instance $I_{X}$ using Theorem 16, for each $X \in \mathcal{F}$. The algorithm outputs yes if and only if there is $X \in \mathcal{F}$, such that $I_{X}$ is a yes instance of RAT MAT CF-BM.

- Theorem 17. The algorithm presented for RAT MAT CF-MM is correct. Moreover, it runs in time $\mathcal{O}\left(2^{(3 \omega+2) k} k^{\mathcal{O}(\log k)}\left\|A_{M}\right\|^{\mathcal{O}(1)} n^{\mathcal{O}(1)}\right)$. 
Proof. Let $\left(G, A_{\mathcal{M}}, k\right)$ be an instance of RAT MAT CF-MM, where matrix $A_{\mathcal{M}}$ represent a matroid $\mathcal{M}=(E, \mathcal{I})$ over field $\mathbb{F}$. Clearly, if the algorithm outputs yes, then indeed $\left(G, A_{\mathcal{M}}, k\right)$ is a yes instance of RAt Mat CF-MM. Next, we argue that if $\left(G, A_{\mathcal{M}}, k\right)$ is a yes instance of RAT MAT CF-MM then the algorithm returns yes. Suppose there is a solution $M \subseteq E(G)$ to RAt MAT CF-MM in $\left(G, A_{\mathcal{M}}, k\right)$. Let $S=\{i, j \mid i j \in M\}$, and $L=\{i \mid$ there is $j \in[n]$ such that $i j \in M$ and $i<j\}$. Observe that $|S|=2 k$. Since $\mathcal{F}$ is an $(n, 2 k)$-universal set, there is $X \in \mathcal{F}$ such that $X \cap S=L$. Note that $S$ is a solution to RAT MAT CF-BM in $I_{X}$. This together with Theorem 16 implies that the algorithm will return yes as the output.

Next, we prove the claimed running time of the algorithm. The algorithm computes $(n, 2 k)$-universal set of size $\mathcal{O}\left(2^{2 k} k^{\mathcal{O}(\log k)} \log n\right)$, in time $\mathcal{O}\left(2^{2 k} k^{\mathcal{O}(\log k)} n \log n\right)$, using Proposition 9. Then for each $X \in \mathcal{F}$, the algorithm creates an instance $I_{X}$ of RAT MAT CF-BM in polynomial time. Furthermore, it resolves the $I_{X}$ of RAT MAT CF-BM in time $\mathcal{O}\left(2^{3 \omega k}\left\|A_{M}\right\|^{\mathcal{O}(1)}\right)$ using Theorem 16. Hence, the running time of the algorithm is bounded by $\mathcal{O}\left(2^{(3 \omega+2) k} k^{\mathcal{O}(\log k)}\left\|A_{M}\right\|^{\mathcal{O}(1)} n^{\mathcal{O}(1)}\right)$.

\subsection{FPT algorithm for Matroid CF-SP}

In this section, we design an FPT algorithm for MATROID CF-SP. The algorithm is based on dynamic programming over representative families. Let $\left(G, s, t, A_{\mathcal{M}}, k\right)$ be an instance of MATRoid CF-SP. Before moving to the description of the algorithm, we need to define some notations. For distinct vertices $u, v \in V(G)$ and an integer $p$, we define: $\mathcal{P}_{u v}^{p}=\{X \subseteq$ $E(G)|| X \mid=p$, there is a $u-v$ path in $G[X]$ containing all edges in $X$, and $X \in \mathcal{I}\}$.

By the definition of convolution of sets, it is easy to see that $\mathcal{P}_{u v}^{p}=\bigcup_{w v \in E(G)} \mathcal{P}_{u w}^{p-1} \star$ $\{\{w v\}\}$. Now we are ready to describe our algorithm for MATroID CF-SP. We aim to store, for each $v \in V(G) \backslash\{s\}, p \leq k$, and $q \leq k-p$, a $q$-representative set $\widehat{\mathcal{P}}_{s v}^{p q}$, of $\mathcal{P}_{s v}^{p}$, of size $\left(\begin{array}{c}p+q \\ q\end{array}\right)$. Notice that for each $v \in V(G) \backslash\{s\}$, we can compute $\mathcal{P}_{s v}^{1}$ in polynomial time, since $\mathcal{P}_{s v}^{1}=\{s v\}$ if $s v \in E(G)$, and is empty otherwise. Moreover, since $\left|\mathcal{P}_{s v}^{1}\right| \leq 1$, therefore, we can set $\widehat{\mathcal{P}}_{s v}^{1 q}=\mathcal{P}_{s v}^{1}$, for each $q \leq k-1$. Next, we iteratively compute, for each $p \in\{2,3, \cdots, k\}$, in increasing order, for each $q \leq k-p$, a $q$-representative $\widehat{\mathcal{P}}_{s v}^{p q}$, of $\mathcal{P}_{s v}^{p}$. From the above discussions, we obtain the following theorem.

Theorem 18. The algorithm Alg-Mat-CF-SP is correct, and runs in time $\mathcal{O}\left(2^{\mathcal{O}(\omega k)} n^{\mathcal{O}(1)}\right)$.

\section{FPT Algorithm for $d$-degenerate Conflict Graphs}

In this section, we show that CF-MM and CF-SP both are in FPT, when the conflict graph $H$ is a $d$-degenerate graphs. These algorithms are based on the notion of independence covering family, which was introduced in [25], defined below.

- Definition 19 ([25]). For a graph $H^{\star}$ and an integer $k$, a $k$-independence covering family, $\mathscr{I}\left(H^{\star}, k\right)$, is a family of independent sets in $H^{\star}$ such that for any independent set $I^{\prime}$ in $H^{\star}$ of size at most $k$, there is a set $I \in \mathscr{I}\left(H^{\star}, k\right)$ such that $I^{\prime} \subseteq I$.

Our algorithms rely on the construction of $k$-independence covering family, for a family of graphs. We first design an algorithm for an annotated version of the CF-MM and CF-SP problems, which we call ANNOTATED CF-MM and ANNOTATED CF-SP, respectively. In the Annotated CF-MM (Annotated CF-SP) problem, the input to CF-MM (CF-SP) is annotated with a $k$-independence covering family $\mathcal{F}$ of $H$. 
Algorithm 1 Alg-CF-MM (Alg-CF-SP).

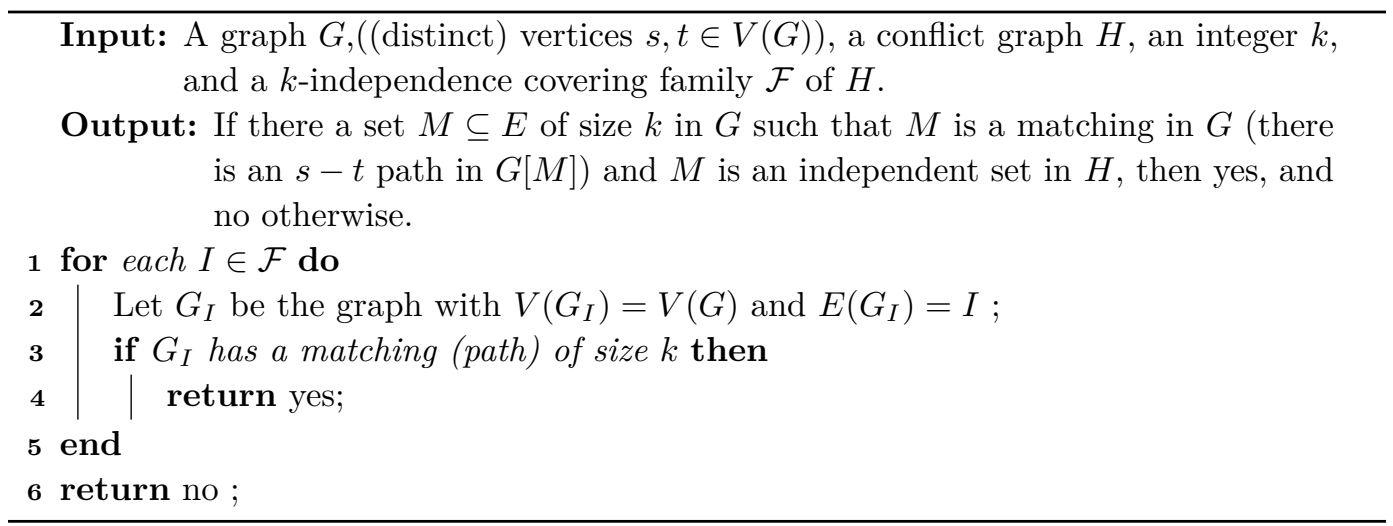

\subsection{Algorithms for Annotated CF-MM and Annotated CF-SP}

In this section, we study the problems Annotated CF-MM and Annotated CF-SP. The algorithm that we design for them run in time polynomial in the size of the input. We give the algorithm Alg-CF-MM (Alg-CF-SP) (Algorithm 1) for AnNotated CF-MM (AnNotated CF-SP).

In the following lemma we prove the correctness of Alg-CF-MM (Alg-CF-SP).

- Lemma 20. The algorithm Alg-CF-MM (Alg-CF-SP) is correct. Moreover, the algorithm runs in time polynomial in the size of the input.

We use Alg-CF-MM (Alg-CF-SP) together with Independence Covering Lemma of [25] to obtain algorithms for CF-MM (CF-SP) when the conflict graph is $d$-degenerate or nowhere dense graph. Towards this we state some lemmata from [25] that we use in our algorithms.

- Proposition 21. [25, Lemma 1.1] There is a randomized algorithm running in polynomial time, that given a d-degenerate graph $H^{\star}$ and an integer $k$ as input, outputs an independent set $I$, such that for every independent set $I^{\prime}$ of size at most $k$ in graph $H^{\star}$, the probability that $I^{\prime} \subseteq I$ is at least $\left(\left(\begin{array}{c}k(d+1) \\ k\end{array}\right) \cdot k(d+1)\right)^{-1}$.

- Proposition 22. [25, Lemmas 3.2 and 3.3] There are two deterministic algorithms $\mathcal{A}_{1}$ and $\mathcal{A}_{2}$, which given a d-degenerate graph $H^{\star}$ and an integer $k$, output independence covering families $\mathscr{I}_{1}\left(H^{\star}, k\right)$ and $\mathscr{I}_{2}\left(H^{\star}, k\right)$, respectively, such that the following conditions are satisfied: i) $\mathcal{A}_{1}$ runs in time $\mathcal{O}\left(\left|\mathscr{I}_{1}\left(H^{\star}, k\right)\right| \cdot(n+m)\right)$, where $\left|\mathscr{I}_{1}\left(H^{\star}, k\right)\right|=\left(\begin{array}{c}k(d+1) \\ k\end{array}\right) \cdot 2^{o(k(d+1))} \cdot \log n$ and ii) $\mathcal{A}_{2}$ runs in time $\mathcal{O}\left(\left|\mathscr{I}_{2}\left(H^{\star}, k\right)\right| \cdot(n+m)\right)$, where $\left|\mathscr{I}_{2}\left(H^{\star}, k\right)\right|=\left(\begin{array}{c}k^{2}(d+1)^{2} \\ k\end{array}\right) \cdot(k(d+$ 1)) $\mathcal{O}(1) \cdot \log n$.

Next, using Proposition 21 and 22, together with Alg-CF-MM (Alg-CF-SP), we obtain randomized and deterministic algorithms, respectively for CF-MM (CF-SP), when the conflict graph is a $d$-degenerate graph.

Theorem 23. There is a randomized algorithm, which given an instance $(G, H, k)$ of $\mathrm{CF}-\mathrm{MM}(\mathrm{CF}-\mathrm{SP})$, where $H$ is a d-degenerate graph, in time $\left(\begin{array}{c}k(d+1) \\ k\end{array}\right) \cdot k(d+1) \cdot n^{\mathcal{O}(1)}$, either reports a failure or correctly outputs that the input is a yes instance of $\mathrm{CF}-\mathrm{MM}(\mathrm{CF}-\mathrm{SP})$. Moreover, if the input is a yes instance of $\mathrm{CF}-\mathrm{MM}(\mathrm{CF}-\mathrm{SP})$, then the algorithm outputs correct answer with a constant probability. 
Proof. Let $(G,(s, t), H, k)$ be an instance CF-MM (CF-SP), where $H$ is a $d$-degenerate graph. We repeat the following procedure $\left(\left(\begin{array}{c}k(1+d) \\ k\end{array}\right) \cdot k(d+1)\right)$ many times: i) the algorithm computes an independent set $I$ in $(H, k)$ using Proposition 21, and ii) the algorithm calls Alg-CF-MM (Alg-CF-SP) with input $(G,(s, t) H, k,\{I\})$

The algorithm outputs yes, if in one of the calls to Alg-CF-MM (Alg-CF-SP), it receives a yes. Otherwise, the algorithm outputs no. The running time analysis of the above procedure follows from Proposition 21 and Lemma 20. Also, given a yes instance, the guarantee on success probability follows from Proposition 21, the number of repetitions, and Lemma 20. Moreover, from Lemma 20 the yes output returned by the algorithm is indeed the correct output to CF-MM(CF-SP)for the given instance. This concludes the proof.

- Theorem 24. CF-MM (CF-SP) admits a deterministic algorithm running in time min $\left\{\left(\begin{array}{c}k(d+1) \\ k\end{array}\right) \cdot 2^{o(k(d+1))} \cdot \log n,\left(\begin{array}{c}k^{2}(d+1)^{2} \\ k\end{array}\right) \cdot(k(d+1))^{\mathcal{O}(1)} \cdot \log n\right\} \cdot n^{\mathcal{O}(1)}$, when the conflict graph is a d-degenerate graph.

Proof. Let $(G,(s, t), H, k)$ be an instance CF-MM (CF-SP), where $H$ is a $d$-degenerate graph. The algorithm starts by computing a $k$-independence covering family $\mathscr{I}(H, k)$ of $H$, using Proposition 22. Next, we call Alg-CF-MM (Alg-CF-SP) with the input $(G,(s, t), H, k$, $\mathscr{I}(H, k))$. The correctness and running time analysis of the above procedure follows from Proposition 22 and Lemma 20. This completes the proof.

\section{Conclusion}

We studied conflict-free (parameterized) variants of MAXIMUM MATCHING (CF-MM) and Shortest PATh (CF-SP). We showed that both CF-MM and CF-SP are W[1]-hard, when parameterized by the solution size. In fact, our W[1]-hardness result for CF-MM holds even when the graph where we want to compute a matching is itself a matching and W[1]-hardness result of CF-SP holds even when the conflict graph is a unit interval graph. Then, we restricted our attention to having conflict graphs belonging to some families of graphs, where the INDEPENDENT SET problem is either polynomial time solvable or solvable in FPT time. In particular, we considered the family of chordal graphs and the family of $d$-degenerate graphs. For the CF-MM problem, we gave an FPT algorithm, when the conflict graph belongs to the family of chordal graphs. We observed that, we cannot obtain an FPT algorithm for the CF-SP problem when the conflict graph is a chordal graph. This holds because unit-interval graphs are chordal, and the problem CF-SP is W[1]-hard, even when the conflict graph is a unit-interval graph. For conflict graphs being $d$-degenerate, we obtained FPT algorithms for both CF-MM and CF-SP. Our results hold even when the conflict graph is a nowhere dense graph. Finally, we studied a variant of CF-MM and CF-SP, where instead of conflicting conditions being imposed by independent sets in a conflict graph, they are imposed by independence constraints in a (representable) matroid. We gave FPT algorithms for the above variant of both CF-MM and CF-SP.

An interesting question is to obtain (parameterized) dichotomy results for CF-MM and CF-SP, based on the families of graphs where the input graphs belong to. Another direction could be studying kernelization complexity for different families of graphs, and also to see what all FPT problems remain FPT with the conflicting constraints. 


\section{References}

1 Akanksha Agrawal, Pallavi Jain, Lawqueen Kanesh, Daniel Lokshtanov, and Saket Saurabh. Conflict Free Feedback Vertex Set: A Parameterized Dichotomy. 43rd International Symposium on Mathematical Foundations of Computer Science, MFCS, pages 53:1-53:15, 2018.

2 Akanksha Agrawal, Pallavi Jain, Lawqueen Kanesh, Pranabendu Misra, and Saket Saurabh. Exploring the Kernelization Borders for Hitting Cycles. 13th International Symposium on Parameterized and Exact Computation, IPEC, pages 14:1-14:14, 2018.

3 Richard Bellman. On a routing problem. Quarterly of applied mathematics, 16(1):87-90, 1958.

4 Marek Cygan, Fedor V. Fomin, Lukasz Kowalik, Daniel Lokshtanov, Dániel Marx, Marcin Pilipczuk, Michal Pilipczuk, and Saket Saurabh. Parameterized Algorithms. Springer, 2015.

5 Andreas Darmann, Ulrich Pferschy, and Joachim Schauer. Determining a minimum spanning tree with disjunctive constraints. International Conference on Algorithmic DecisionTheory (ADT), pages 414-423, 2009.

6 Andreas Darmann, Ulrich Pferschy, Joachim Schauer, and Gerhard J. Woeginger. Paths, trees and matchings under disjunctive constraints. Discrete Applied Mathematics, 159(16):1726-1735, 2011.

7 Edsger W Dijkstra. A note on two problems in connexion with graphs. Numerische mathematik, 1(1):269-271, 1959.

8 Rodney G. Downey and Michael R. Fellows. Fixed Parameter Tractability and Completeness. Complexity Theory: Current Research, pages 191-225, 1992.

9 Rodney G. Downey and Michael R. Fellows. Fundamentals of Parameterized Complexity. Texts in Computer Science. Springer, 2013.

10 Leah Epstein, Lene M. Favrholdt, and Asaf Levin. Online variable-sized bin packing with conflicts. Discrete Optimization, 8(2):333-343, 2011.

11 Guy Even, Magnús M. Halldórsson, Lotem Kaplan, and Dana Ron. Scheduling with conflicts: online and offline algorithms. Journal of Scheduling, 12(2):199-224, 2009.

12 Shimon Even and Oded Kariv. An $O\left(n^{2.5}\right)$ algorithm for maximum matching in general graphs. Foundations of Computer Science (FOCS), pages 100-112, 1975.

13 J. Flum and M. Grohe. Parameterized Complexity Theory (Texts in Theoretical Computer Science. An EATCS Series). Springer-Verlag, Secaucus, NJ, USA, 2006.

14 Fedor V. Fomin, Daniel Lokshtanov, Fahad Panolan, and Saket Saurabh. Efficient Computation of Representative Families with Applications in Parameterized and Exact Algorithms. Journal of the ACM, 63(4):29:1-29:60, 2016.

15 Fedor V Fomin, Daniel Lokshtanov, and Saket Saurabh. Efficient computation of representative sets with applications in parameterized and exact algorithms. Symposium on Discrete Algorithms (SODA), pages 142-151, 2014.

16 Harold N. Gabow, Shachindra N Maheshwari, and Leon J. Osterweil. On two problems in the generation of program test paths. IEEE Transactions on Software Engineering, 2(3):227-231, 1976.

17 Fǎnică Gavril. The intersection graphs of subtrees in trees are exactly the chordal graphs. Journal of Combinatorial Theory, Series B, 16(1):47-56, 1974.

18 Michel Gendreau, Gilbert Laporte, and Frédéric Semet. Heuristics and lower bounds for the bin packing problem with conflicts. Computers \& OR, 31(3):347-358, 2004.

19 Pallavi Jain, Lawqueen Kanesh, and Pranabendu Misra. Conflict Free Version of Covering Problems on Graphs: Classical and Parameterized. Computer Science - Theory and Applications - 13th International Computer Science Symposium in Russia (CSR), pages 194-206, 2018.

20 Klaus Jansen. An approximation scheme for bin packing with conflicts. Journal of combinatorial optimization, 3(4):363-377, 1999.

21 Minghui Jiang. On the parameterized complexity of some optimization problems related to multiple-interval graphs. Theoretical Computer Science, 411(49):4253-4262, 2010.

22 Ton Kloks. Treewidth, Computations and Approximations, volume 842 of Lecture Notes in Computer Science. Springer, 1994. 
23 KW Krause, MA Goodwin, and RW Smith. Optimal software test planning through automated network analysis. TRW Systems Group, 1973.

24 Daniel Lokshtanov, Pranabendu Misra, Fahad Panolan, and Saket Saurabh. Deterministic truncation of linear matroids. International Colloquium on Automata, Languages, and Programming (ICALP), pages 922-934, 2015.

25 Daniel Lokshtanov, Fahad Panolan, Saket Saurabh, Roohani Sharma, and Meirav Zehavi. Covering small independent sets and separators with applications to parameterized algorithms. Symposium on Discrete Algorithms (SODA), pages 2785-2800, 2018.

26 Dániel Marx. A parameterized view on matroid optimization problems. Theoretical Computer Science, 410(44):4471-4479, 2009.

27 Dániel Marx. A parameterized view on matroid optimization problems. Theoretical Computer Science, 410(44):4471-4479, 2009.

28 Silvio Micali and Vijay V Vazirani. An $O(\sqrt{\mid} V|| E \mid)$ algorithm for finding maximum matching in general graphs. Foundations of Computer Science (FOCS), pages 17-27, 1980.

29 Moni Naor, Leonard J Schulman, and Aravind Srinivasan. Splitters and near-optimal derandomization. Foundations of Computer Science (FOCS), pages 182-191, 1995.

30 Rolf Niedermeier. Invitation to Fixed-Parameter Algorithms, volume 31 of Oxford Lecture Series in Mathematics and its Applications. Oxford University Press, Oxford, 2006.

31 James G Oxley. Matroid theory, volume 3. Oxford University Press, 2006.

32 Ulrich Pferschy and Joachim Schauer. The Knapsack Problem with Conflict Graphs. Journal of Graph Algorithms and Applications, 13(2):233-249, 2009.

33 Ulrich Pferschy and Joachim Schauer. The maximum flow problem with conflict and forcing conditions. Network Optimization, pages 289-294, 2011.

34 Ulrich Pferschy and Joachim Schauer. The maximum flow problem with disjunctive constraints. Journal of Combinatorial Optimization, 26(1):109-119, 2013.

35 Ulrich Pferschy and Joachim Schauer. Approximation of knapsack problems with conflict and forcing graphs. Journal of Combinatorial Optimization, 33(4):1300-1323, 2017.

36 Virginia Vassilevska Williams. Multiplying Matrices Faster Than Coppersmith-winograd. Symposium on Theory of Computing (STOC), pages 887-898, 2012. 